\title{
If it feels like Myxoedema Coma, then it probably is!
}

\author{
Kang $\mathrm{H}$, Bashari W, Oyibo SO \\ Department of Endocrinology, Peterborough City Hospital, Peterborough, UK
}

\section{Introduction}

Myxoedema coma (hypothyroid crisis) is an endocrine emergency where patients are in a severe hypothyroid state. It is a life-threatening and potentially reversible condition, but can be difficult to recognise due to the varied symptomatology. Elderly female patients are at highest risk and infective illnesses and septicaemia are the main precipitants. ${ }^{1}$ The mainstay of treatment is intravenous administration of thyroid hormones (L-Tri-iodothyronine) and supportive treatment. Despite our immense understanding of this emergency, hypothyroid crisis remains widely under-appreciated.

Hypothyroid crisis occurring in the presence of mildly abnormal thyroid function test (TFT) results is a rare but recognised phenomenon. ${ }^{2}$

\section{Case}

An 82 year-old lady was admitted to hospital with a simple urinary tract infection with a surprisingly poor response to therapy. She has hypothyroidism secondary to a thyroidectomy 8 years ago usually well-controlled on oral levothyroxine $50 \mathrm{mcg}$ daily and chronic mild hyponatraemia due to inappropriate anti-diuretic hormone secretion (with previously normal adrenal function). Her condition worsened rapidly with clinical features suggestive of sepsis. Despite fluid resuscitation and antibiotics, she became unconsciousness with hypotension, relative bradycardia, hypothermia, hypoxaemia: clinical features suggestive of a severe hypothyroid crisis.

\section{Investigations and Treatment}

Her biochemical test results are shown in the Table 1. Despite mildly hypothyroid TFT results, but features suggesting hypothyroid crisis, she was given a trial of intravenous L-Tri-iodothyronine $\left(\mathrm{T}_{3}\right)$ at a dose of 50 mcg initially followed by $25 \mathrm{mcg} 8$ hourly. ${ }^{3}$ She was also given intravenous steroids. She showed a striking clinical response just after two doses: in fact after the first dose she gained full consciousness within 4 hours.

\begin{tabular}{|c|c|c|}
\hline Tabl & & \\
\hline Test & Results & $\begin{array}{l}\text { Normal } \\
\text { values }\end{array}$ \\
\hline $\begin{array}{c}\text { Thyroid stimulating hormone, } \\
\text { TSH }(\mathrm{mU} / \mathrm{L})\end{array}$ & 6.37 & $0.3-4.2$ \\
\hline Free $T_{3}(\mathrm{pmol} / \mathrm{L})$ & 3.2 & $3.1-6.8$ \\
\hline Free $T_{4}(\mathrm{pmol} / \mathrm{L})$ & 21.5 & $12.0-22.0$ \\
\hline Random Cortisol (nmol/L) & 311 & $\begin{array}{l}\text { Done 2 days } \\
\text { after } \\
\text { admission }\end{array}$ \\
\hline Random Glucose (mmol/L) & 7.1 & $<7.0$ \\
\hline Haemoglobin (g/dL) & 12.9 & $115-165$ \\
\hline Platelets $\left(10^{9} / \mathrm{L}\right)$ & 140 & $150-400$ \\
\hline White cell count $\left(10^{9} / \mathrm{L}\right)$ & 6.7 & $4.0-11.0$ \\
\hline C-reactive protein (mg/L) & 12 & $<10$ \\
\hline Troponin-T (ng/L) & 14 & $<14$ \\
\hline Sodium $(\mathrm{mmol} / \mathrm{L})$ & 120 & $133-146$ \\
\hline Potassium (mmol/L) & 3.7 & $3.5-5.3$ \\
\hline Chloride $(\mathrm{mmol} / \mathrm{L})$ & 87 & $95-108$ \\
\hline Adjusted Calcium (mmol/L) & 2.19 & $2.2-2.6$ \\
\hline Creatinine $(\mu \mathrm{mol} / \mathrm{L})$ & 88 & $50-120$ \\
\hline Urea $(\mathrm{mmol} / \mathrm{L})$ & 6.0 & $2.5-7.8$ \\
\hline eGFR $(\mathrm{mL} / \mathrm{min})$ & 53 & $>60$ \\
\hline Amylase (U/L) & 43 & $0-100$ \\
\hline Coagulation (INR) & 0.90 & $0.8-1.25$ \\
\hline Lactate $(\mathrm{mmol} / \mathrm{L})$ & 2.20 & $0.6-2.5$ \\
\hline
\end{tabular}

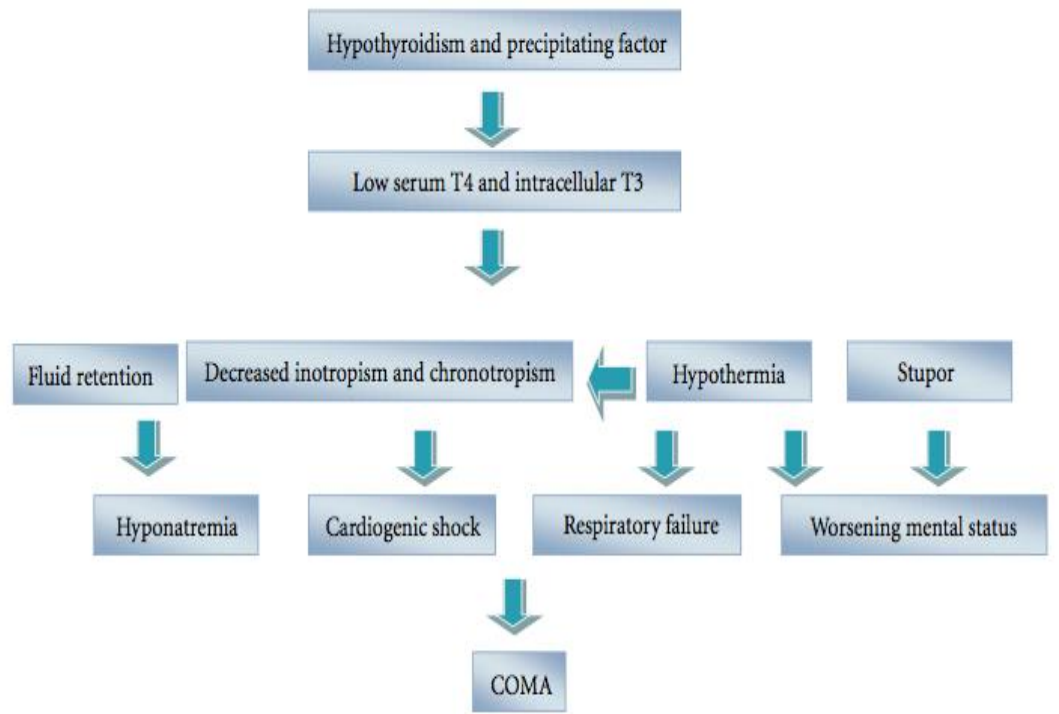

Figure 2 - Pathogenesis of myxoedema coma adapted from Mathew et al ${ }^{1}$

- Intracellular deficiency of T3 in the presence of mild hypothyroidism can be exacerbated by the increased metabolic demand of infections and septicaemia.

- This patient's TSH levels have always been below $2.5 \mathrm{mU} / \mathrm{l}$ until her admission when it was $6.37 \mathrm{mU} / \mathrm{l}$.

- One should always be aware of the probability of co-existing adrenal crisis.

\section{Conclusion}

Our patient was soon discharged, remains well and is under regular follow-up. This case emphasises the potential reversibility of this life-threatening emergency. A low index of clinical suspicion amidst multiple co-morbidities and sepsis is required, even in the face of near normal or mildly hypothyroid TFT results. 